\title{
On the first secondary invariant of Molino's central sheaf
}

\author{
by Jesús A. Álvarez López (Lugo)
}

\begin{abstract}
For a Riemannian foliation on a closed manifold, the first secondary invariant of Molino's central sheaf is an obstruction to tautness. Another obstruction is the class defined by the basic component of the mean curvature with respect to some metric. Both obstructions are proved to be the same up to a constant, and other geometric properties are also proved to be equivalent to tautness.
\end{abstract}

1. Introduction and main results. Let $\mathcal{F}$ be a Riemannian foliation on a closed manifold $M[19], \Omega(M / \mathcal{F})$ its basic complex, and $H^{\cdot}(M / \mathcal{F})$ its basic cohomology $[6,7,13]$. There is a locally trivial sheaf $\mathcal{C}=\mathcal{C}(\mathcal{F})$ of Lie algebras of germs of transverse Killing fields whose "transverse orbits" are the leaf closures [16, 17]. It is called the central sheaf of $\mathcal{F}$. The typical fiber of $\mathcal{C}$ is the opposite of the structural Lie algebra $\mathfrak{g}$ of $\mathcal{F}$. The sheaf $\mathcal{C}$ canonically defines a vector bundle $C=C(\mathcal{F})$ over $M$ with a flat connection. The corresponding multiplicative homomorphism $\Delta_{*}: H^{\cdot}(\operatorname{gl}(q), \mathrm{O}(q)) \rightarrow H^{\cdot}(M)[12], q=\operatorname{codim} \mathcal{F}$, can be given as the composite of a homomorphism $\Delta_{*}=\Delta(\mathcal{F})_{*}: H^{\cdot}(\operatorname{gl}(q), \mathrm{O}(q)) \rightarrow H^{\cdot}(M / \mathcal{F})$ and the canonical homomorphism $H^{\cdot}(M / \mathcal{F}) \rightarrow H^{\cdot}(M)$. We get basic secondary invariants $\Delta_{*}\left(y_{i}\right)=\Delta(\mathcal{F})_{*}\left(y_{i}\right) \in H^{2 i-1}(M / \mathcal{F}), i=1, \ldots, 2[(m+1) / 2]-1$, $m=\operatorname{dim} \mathfrak{g}$. The basic class $\Delta_{*}\left(y_{1}\right)$ will be studied in this paper. It would also be interesting to study the geometric information contained in the $\Delta_{*}\left(y_{i}\right)$ for $i>1$.

It was pointed out in [18] that, for Riemannian flows, $\Delta_{*}\left(y_{1}\right)$ is the obstruction to tautness; i.e. the obstruction to the existence of a metric on $M$ such that the leaves are minimal submanifolds. This property also holds for $\mathcal{F}$ of arbitrary dimension: Suppose $\mathcal{F}$ is transversely orientable for simplicity; then $\mathcal{F}$ is taut if and only if $H^{q}(M / \mathcal{F}) \neq 0$, which is equivalent

1991 Mathematics Subject Classification: 53C12, 57R30.

Key words and phrases: foliation, taut.

Supported in part by Spanish DGICYT grant PB90-0765. 
to the triviality of the sheaf $\bigwedge^{m} \mathcal{C}$ [22], and this in turn is equivalent to $\Delta_{*}\left(y_{1}\right)=0$. There is another obstruction to tautness: For any bundle-like metric, the basic component of the mean curvature form of the leaves is closed and defines a class $\xi=\xi(\mathcal{F}) \in H^{1}(M / \mathcal{F})$, which depends only on $\mathcal{F}$ and vanishes if and only if $\mathcal{F}$ is taut [2]. We close this circle of ideas by proving directly that both obstructions are the same up to a constant:

TheOREM 1.1. With the above notation, $\xi=-2 \pi \Delta_{*}\left(y_{1}\right)$.

We also prove other relations between $\Delta_{*}\left(y_{1}\right)$ and geometric properties of $\mathcal{F}$. Consider the filtration of $\Omega^{\cdot}(M / \mathcal{F})$ given by the differential ideals $F^{k} \Omega(M / \mathcal{F})$, where an $\alpha \in \Omega^{r}(M / \mathcal{F})$ is in $F^{k} \Omega^{r}(M / \mathcal{F})$ if $i_{X} \alpha=0$ for $X=X_{1} \wedge \ldots \wedge X_{r-k+1}$ with the vector fields $X_{j}$ tangent to the leaf closures. The corresponding spectral sequence $\left(E_{i}, d_{i}\right)$ converges to $H^{\cdot}(M / \mathcal{F})$ (cf. [8, $\S 2])$. If $M / \overline{\mathcal{F}}$ denotes the space of leaf closures of $\mathcal{F}$, there is a canonical isomorphism $E_{2}^{\cdot, 0} \cong H^{\cdot}(M / \overline{\mathcal{F}})$. So there is a canonical injection $H^{1}(M / \overline{\mathcal{F}}) \rightarrow$ $H^{1}(M / \mathcal{F})$. Let $F^{k} H^{\cdot}(M / \mathcal{F})$ be the induced filtration of $H^{\cdot}(M / \mathcal{F})$. The element defined by $\Delta_{*}\left(y_{1}\right)$ in $H^{1}(M / \mathcal{F}) / F^{1} H^{1}(M / \mathcal{F}) \equiv E_{0}^{0,1}$ will be denoted by $\bar{\Delta}_{*}\left(y_{1}\right)$. Thus $\bar{\Delta}_{*}\left(y_{1}\right)=0$ if and only if $\Delta_{*}\left(y_{1}\right) \in F^{1} H^{1}(M / \mathcal{F}) \equiv E_{\infty}^{1,0} \cong$ $H^{1}(M / \overline{\mathcal{F}})$.

THEOREM 1.2. With the above notation, $\bar{\Delta}_{*}\left(y_{1}\right)=0$ if and only if $\mathfrak{g}$ is unimodular.

THEOREM 1.3. With the above notation, suppose $\mathcal{F}$ admits a transverse parallelism. Let $\mathcal{H}$ be any representative of the holonomy pseudogroup of $\mathcal{F}$ on some manifold $T$. Then:

(i) If $\mathfrak{g}$ is unimodular, then $\Delta_{*}\left(y_{1}\right)=0$ if and only if the $\mathcal{H}$-orbit closures are minimal submanifolds for some $\mathcal{H}$-invariant metric on $T$.

(ii) If $\mathfrak{g}$ is not unimodular, then the $\mathcal{H}$-orbit closures are minimal submanifolds for some $\mathcal{H}$-invariant metric on $T$.

Thus $\mathcal{F}$ is taut if and only if $\mathfrak{g}$ is unimodular and the $\mathcal{H}$-orbit closures are minimal submanifolds for some $\mathcal{H}$-invariant metric.

If $\mathcal{F}$ does not admit any transverse parallelism, a similar result can be stated by considering the horizontal lifting $\widehat{\mathcal{F}}$ to the principal bundle of transverse orthonormal frames for some fixed transverse Riemannian structure $[16,17]$. In particular, we have the following.

COROLLARY 1.4. With the above notation, let $\widehat{\mathcal{H}}$ be the holonomy pseudogroup of $\widehat{\mathcal{F}}$. Then $\mathcal{F}$ is taut if and only if $\mathfrak{g}$ is unimodular and the $\widehat{\mathcal{H}}$-orbit closures are minimal submanifolds for some $\widehat{\mathcal{H}}$-invariant metric.

For a bundle-like metric on $M$, let $\kappa$ be the mean curvature form of the leaves, and $\kappa_{\mathrm{b}}$ its basic component. 
THEOREM 1.5. With the above notation, the following holds:

(i) If $\mathfrak{g}$ is unimodular then, for any bundle-like metric, $\kappa_{\mathrm{b}}$ vanishes on vectors tangent to the leaf closures.

(ii) If $\mathfrak{g}$ is not unimodular, then there is a bundle-like metric such that $\kappa_{\mathrm{b}}$ vanishes on vectors orthogonal to the leaf closures.

This theorem can be sharpened by the recent result of D. Domínguez [5], showing the existence of a bundle-like metric on $M$ with basic mean curvature of the leaves. Indeed any representative of $\xi$ can be realized as the mean curvature for some bundle-like metric. For Lie foliations with dense leaves, the result is very explicit:

COROLlary 1.6. If $\mathcal{F}$ is a Lie $\mathfrak{g}$-foliation with dense leaves then, for any bundle-like metric, the basic component of the mean curvature corresponds to the trace of the adjoint representation by the canonical identity $\Omega \cdot(M / \mathcal{F}) \equiv$ $\wedge \mathfrak{g}^{*}$. Moreover, such a form can always be realized as the mean curvature for some bundle-like metric.

These results depend only on the holonomy pseudogroup of $\mathcal{F}$. Thus, with slightly more generality, we shall consider a complete pseudogroup $\mathcal{H}$ of local isometries of a Riemannian manifold $T[10,11]$.

2. A remark on the first secondary characteristic class of a flat vector bundle. Let $(M, g)$ be a Riemannian manifold, $\varrho: V \rightarrow M$ a rank $m$ vector bundle with a flat connection $\nabla, P$ the $\operatorname{Gl}(m)$-principal bundle of frames of $V$, and $\omega$ the connection form defined by $\nabla$. The induced connection on $\bigwedge V$ will also be denoted by $\nabla$. For any given $\mathrm{O}(m)$-reduction of $P$ defined by a section $s: M \rightarrow P / \mathrm{O}(m)$, we have the multiplicative homomorphism $\Delta_{*}: H(\mathrm{gl}(m), \mathrm{O}(m)) \rightarrow H^{\cdot}(M)[12$, Theorem 4.43], yielding secondary characteristic invariants $\Delta_{*}\left(y_{i}\right) \in H^{2 i-1}(M)$ for $i=1, \ldots, 2[(m+$ $1) / 2]-1\left[12\right.$, Theorem 6.33]. A representative of $\Delta_{*}\left(y_{1}\right)$ is the form $\Delta\left(y_{1}\right)=$ $\frac{1}{2 \pi} s^{*}($ trace $\omega) \in \Omega^{1}(M)[12$, Proof of Proposition 6.34].

Recall that any smooth section $X$ of $\bigwedge V$ canonically defines a smooth section $\widehat{X}$ of $\varrho^{*} \bigwedge V \equiv \bigwedge \varrho^{*} V$. Identifying $\varrho^{*} V$ with the vertical bundle of $\varrho$ in the canonical way, we can consider such an $\widehat{X}$ as a smooth section of $\bigwedge \mathrm{T} V$ over $V$. Moreover, if $\widetilde{Z}$ is the horizontal lifting of any vector field $Z$ on $M$, we get

$$
\widehat{\nabla_{Z} X}=\theta_{\tilde{Z}} \widehat{X}
$$

where $\theta_{\tilde{Z}}$ denotes the Lie derivative with respect to $\widetilde{Z}$. This can be seen as follows. The parallel transport along the integral curves of $Z$ is given by the integral curves of $\widetilde{Z}$. In particular, the restriction of the flow of $\widetilde{Z}$ between two fibers of $\varrho$ is linear, and thus can be canonically identified with 
its derivative at each point. Therefore (1) follows from the usual expression of the covariant derivative in terms of parallel transport and the usual expression of the Lie derivative in terms of the flow of vector fields.

Consider the Riemannian structure on $V$ defined by the $\mathrm{O}(m)$-reduction of $P$. We get an induced Riemannian structure on the vertical bundle of $\varrho$ by identifying it with $\varrho^{*} V$. Let $\widehat{g}$ be the Riemannian metric on $V$ defined as the orthogonal sum of the lift of $g$ to the horizontal bundle and the Riemannian structure on the vertical bundle. The $\widehat{g}$-mean curvature form of the fibers of $\varrho$ will denoted by $\kappa_{V}$. The induced metric on $\bigwedge \mathrm{T} V$ will also be denoted by $\widehat{g}$.

Proposition 2.1. $\kappa_{V}=2 \pi \varrho^{*} \Delta\left(y_{1}\right)$.

Pr o of. We can clearly assume $V$ is an oriented vector bundle. So $P$ has a $\mathrm{Gl}^{+}(m)$-reduction $P^{+}$. Consider the homomorphism det $: \mathrm{Gl}^{+}(m) \rightarrow \mathbb{R}^{+}$, and the corresponding bundle map $P^{+} \rightarrow \bar{P}=P^{+} \times_{\mathrm{Gl}^{+}(m)} \mathbb{R}^{+}$. Then $\nabla$ defines a flat connection on $\bar{P}$, and let $\bar{\omega}$ be its connection form.

The section $s$ defines a section $\bar{s}$ of $\bar{P} \rightarrow M$ because the composite

$$
\mathrm{SO}(m) \hookrightarrow \mathrm{Gl}^{+}(m) \stackrel{\text { det }}{\longrightarrow} \mathbb{R}^{+}
$$

is trivial. By functoriality of the construction of the characteristic homomorphism under homomorphisms of structural groups [12, Theorem 4.43(iii)], we have

$$
\bar{s}^{*} \bar{\omega}=2 \pi \Delta\left(y_{1}\right) .
$$

(See the proof of Proposition 6.34 in [12].)

Since $V$ is an oriented bundle, there is a non-vanishing section $X \in$ $C^{\infty}\left(\bigwedge^{m} V\right)$, with a corresponding section $\widehat{X} \in C^{\infty}\left(\varrho^{*} \bigwedge^{m} V\right) \equiv C^{\infty}\left(\bigwedge^{m} \varrho^{*} V\right)$. By identifying $\varrho^{*} V$ with the vertical bundle, if $X$ is unitary, then $\chi=\widehat{g}(\widehat{X}, \cdot)$ is the characteristic form of the fibers of $\varrho[20]$; i.e. $\chi(U)=\widehat{g}(\widehat{X}, U)$ for any $U \in C^{\infty}(\bigwedge \mathrm{T} V)$. Thus

$$
\theta_{Y} \widehat{X}=\kappa_{V}(Y) \widehat{X}
$$

for any horizontal $\varrho$-projectable vector field $Y$ on $V$. Indeed, $\theta_{Y} \widehat{X}=f \widehat{X}$ for some function $f$ on $V$ because the flow of $Y$ maps fibers of $\varrho$ to fibers of $\varrho$, and Rummler's mean curvature formula implies [20]

$$
0=\theta_{Y}(\chi(\widehat{X}))=\left(\theta_{Y} \chi\right)(\widehat{X})+\chi\left(\theta_{Y}(\widehat{X})\right)=-\kappa_{V}(Y)+f .
$$

On the other hand, $\bar{P}$ can be canonically identified with the principal bundle of oriented frames of the line bundle $\bigwedge^{m} V$. Thus

$$
\nabla_{Z} X=\left(\bar{s}^{*} \bar{\omega}\right)(Z) X
$$

for any vector field $Z$ on $M$, where $\widetilde{Z}$ is its horizontal lifting. 
Therefore

$$
\begin{aligned}
\kappa_{V}(\widetilde{Z}) \widehat{X} & =\theta_{\tilde{Z}} \widehat{X} & & \text { by }(3) \\
& =\widehat{\nabla_{Z} X} & & \text { by }(1) \\
& =\left(\bar{s}^{*} \bar{\omega}\right)(Z) \widehat{X} & & \text { by }(4) \\
& =2 \pi \varrho^{*} \Delta\left(y_{1}\right)(\widetilde{Z}) \widehat{X} & & \text { by }(2) .
\end{aligned}
$$

The result now follows because $\kappa_{V}$ and $\varrho^{*} \Delta\left(y_{1}\right)$ vanish on vertical vectors.

3. Preliminaries on complete pseudogroups of local isometries. Let $\mathcal{H}$ be a complete pseudogroup of local isometries of a Riemannian manifold $(T, g), T / \mathcal{H}$ the space of $\mathcal{H}$-orbits, and $\overline{\mathcal{H}}$ the closure of $\mathcal{H}$ [10]. Thus $T / \overline{\mathcal{H}}$ is the space of $\mathcal{H}$-orbit closures.

If $\mathcal{H}$ preserves a parallelism on $T$, then we have the following description due to E. Salem [21]. The space $T / \overline{\mathcal{H}}$ is a manifold and the canonical projection $\pi_{\mathrm{b}}: T \rightarrow T / \overline{\mathcal{H}}$ is a submersion. Moreover, for some Lie group $G$ and some dense subgroup $\Lambda \subset G$, every point in $T / \overline{\mathcal{H}}$ has a neighborhood $U$ so that the restriction of $\mathcal{H}$ to $\pi_{\mathrm{b}}^{-1}(U)$ is equivalent to the pseudogroup generated by the action of $\Lambda$ on $G \times U$, acting by left multiplication on $G$ and trivially on $U$. Furthermore, $\pi_{\mathrm{b}}$ corresponds to the canonical second projection of $G \times U$ onto $U$ by this equivalence. The Lie algebra $\mathfrak{g}$ of $G$ is called the structural Lie algebra of $\mathcal{H}$, and $\pi_{\mathrm{b}}$ its basic projection.

For arbitrary $\mathcal{H}$, it is standard to consider the $\mathrm{O}(n)$-principal bundle $\pi$ : $\widehat{T} \rightarrow T$ of orthonormal frames on $T$ with the Levi-Civita connection, where $n=\operatorname{dim} T$, and the complete pseudogroup $\widehat{\mathcal{H}}$ canonically defined by $\mathcal{H}$ on $\widehat{T}$. The canonical parallelisms on $\widehat{T}$ are $\widehat{\mathcal{H}}$-invariant, thus Salem's description holds for $\widehat{\mathcal{H}}$. The structural Lie algebra of $\widehat{\mathcal{H}}$ is also called the structural Lie algebra of $\mathcal{H}$. (There is no ambiguity when $\mathcal{H}$ preserves a parallelism.) The $\mathrm{O}(n)$-action on $\widehat{T}$ preserves $\widehat{\mathcal{H}}$, and thus there is an induced $\mathrm{O}(n)$-action on the manifold $W$ of $\widehat{\mathcal{H}}$-orbit closures so that the basic projection $\pi_{\mathrm{b}}$ is $\mathrm{O}(n)$-equivariant, yielding a canonical identity

$$
T / \overline{\mathcal{H}} \equiv W / \mathrm{O}(n) \text {. }
$$

The complex of $\mathcal{H}$-invariant differential forms will be denoted by $\Omega_{\mathcal{H}}=$ $\Omega(T)_{\mathcal{H}}$, and its cohomology by $H^{\cdot}(T)_{\mathcal{H}}$. We shall also use the notation $\Omega_{\mathcal{H}, i=0}$ for the space of $\mathcal{H}$-invariant forms which vanish on vector fields tangent to the $\mathcal{H}$-orbit closures. Similarly, let $\Omega(W)_{\mathrm{O}(n), i=0}$ be the complex of $\mathrm{O}(n)$-invariant differential forms on $W$ which vanish on vector fields tangent to the $\mathrm{O}(n)$-orbits.

Define a filtration of $\Omega_{\mathcal{H}}$ by differential ideals $F^{k} \Omega_{\mathcal{H}}$, where an $\mathcal{H}$ invariant $r$-form $\alpha$ is in $F^{k} \Omega_{\mathcal{H}}^{r}$ if $i_{X} \alpha=0$ for $X=X_{1} \wedge \ldots \wedge X_{r-k+1}$ 
with the vector fields $X_{j}$ tangent to the orbit closures. The corresponding spectral sequence $\left(E_{i}, d_{i}\right)=\left(E_{i}(\mathcal{H}), d_{i}\right)$ converges to $H^{\cdot}(T)_{\mathcal{H}}$. We have $E_{0}^{u, \cdot}=F^{u} \Omega_{\mathcal{H}} / F^{u+1} \Omega_{\mathcal{H}}$ with the differential map induced by the de Rham derivative, and thus

$$
E_{1}^{u, v}=\frac{F^{u} \Omega_{\mathcal{H}}^{v} \cap d^{-1}\left(F^{u+1} \Omega_{\mathcal{H}}^{v+1}\right)}{F^{u+1} \Omega_{\mathcal{H}}^{v}+d\left(F^{u} \Omega_{\mathcal{H}}^{v-1}\right)} .
$$

Since clearly $F^{u} \Omega_{\mathcal{H}}^{u}=\Omega_{\mathcal{H}, i=0}^{u}$, we get $E_{0}^{r, 0} \equiv \Omega_{\mathcal{H}, i=0}$. Moreover,

$$
\theta_{X}\left(\Omega_{\mathcal{H}, i=0}\right)=0
$$

if the vector field $X$ is tangent to the $\mathcal{H}$-orbit closures. Indeed, it is easy to check that (6) follows if it is proved for $\widehat{\mathcal{H}}$. But since $\widehat{\mathcal{H}}$ preserves a parallelism, it is enough to prove (6) for $\widehat{\mathcal{H}}$-invariant functions, and it is obvious in this case since such functions are constant on the $\widehat{\mathcal{H}}$-orbit closures.

From (6) we get $i_{X} d\left(\Omega_{\mathcal{H}, i=0}\right)=0$ for such $X$; i.e. $d\left(\Omega_{\mathcal{H}, i=0}\right) \subset \Omega_{\mathcal{H}, i=0}$, yielding

$$
E_{1}^{;, 0} \equiv \Omega_{\mathcal{H}, i=0} \cong \Omega(W)_{\mathrm{O}(n), i=0},
$$

where the isomorphism is given by $\alpha \mapsto \bar{\alpha}$ if $\pi^{*} \alpha=\pi_{\mathrm{b}}^{*} \bar{\alpha}$. Therefore, from (5) and the result of [23] we get

$$
E_{2}^{\cdot, 0} \cong H^{\cdot}(T / \overline{\mathcal{H}}) \text {. }
$$

Now, from the general theory of spectral sequences, there is a canonical injection $E_{2}^{1,0} \rightarrow H^{1}(T)_{\mathcal{H}}$. So (8) yields an injection $H^{1}(T / \overline{\mathcal{H}}) \rightarrow H^{1}(T)_{\mathcal{H}}$.

If $F^{k} H^{\cdot}(T)_{\mathcal{H}}$ denotes the filtration of $H^{\cdot}(T)_{\mathcal{H}}$ induced by the filtration of $\Omega_{\mathcal{H}}$, then $E_{\infty}^{0,1} \equiv H^{1}(T)_{\mathcal{H}} / F^{1} H^{1}(T)_{\mathcal{H}}$ and $E_{\infty}^{1,0} \equiv F^{1} H^{1}(T)_{\mathcal{H}} \cong H^{1}(T / \overline{\mathcal{H}})$ by (8). Moreover, $\pi_{\infty}^{*}: E_{\infty}^{0,1}(\mathcal{H}) \rightarrow E_{\infty}^{0,1}(\widehat{\mathcal{H}})$ is injective since $\pi^{*}: H^{1}(T)_{\mathcal{H}} \rightarrow$ $H^{1}(\widehat{T})_{\widehat{\mathcal{H}}}$ is easily checked to be injective with usual arguments involving the standard spectral sequence defined by $\pi$.

A vector bundle $\varrho: V \rightarrow T$ will be called an $\mathcal{H}$-vector bundle if, for any diffeomorphism $h: U_{1} \rightarrow U_{2}$ in $\mathcal{H}$, there is a vector bundle homomorphism $\widetilde{h}: \varrho^{-1}\left(U_{1}\right) \rightarrow \varrho^{-1}\left(U_{2}\right)$ over $h$ satisfying $\widetilde{\mathrm{id}}_{T}=\operatorname{id}_{V}, \widetilde{h_{1} h_{2}}=\widetilde{h}_{1} \widetilde{h}_{2}$, and $\left.\widetilde{h}\right|_{\varrho^{-1}(U)}=\left.\widetilde{h}\right|_{U}$ for every open subset $U \subset \operatorname{dom} h$. A connection on $V$ will be called an $\mathcal{H}$-connection if it is invariant by the $\widetilde{h}$. The following is a natural example of an $\mathcal{H}$-vector bundle with an $\mathcal{H}$-flat connection. The locally trivial sheaf of infinitesimal transformations of $\overline{\mathcal{H}}$ will be denoted by $\mathcal{C}=\mathcal{C}(\mathcal{H})[21]$. Such a $\mathcal{C}$ is a sheaf of Lie algebras, whose typical fiber is the opposite Lie algebra $\mathfrak{g}^{-}$of $\mathfrak{g}$. The corresponding $\mathcal{H}$-vector bundle will be denoted by $C=C(\mathcal{H})$, and the corresponding $\mathcal{H}$-flat connection by $\nabla$. By naturality, the multiplicative homomorphism $\Delta_{*}: H^{\cdot}(\operatorname{gl}(n), \mathrm{O}(n)) \rightarrow H^{\cdot}(T)$ defined by $\nabla$ is the composite of a multiplicative homomorphism $\Delta_{*}=\Delta(\mathcal{H})_{*}$ : $H^{\cdot}(\operatorname{gl}(n), \mathrm{O}(n)) \rightarrow H^{\cdot}(T)_{\mathcal{H}}$ and the canonical homomorphism $H^{\cdot}(T)_{\mathcal{H}} \rightarrow$ 
$H^{\cdot}(T)$. This yields secondary characteristic invariants $\Delta_{*}\left(y_{i}\right)=\Delta(\mathcal{H})_{*}\left(y_{i}\right) \in$ $H^{2 i-1}(T)_{\mathcal{H}}$. Indeed, the representatives defined in [12] are $\mathcal{H}$-invariant; in particular, $\Delta\left(y_{1}\right) \in \Omega_{\mathcal{H}}^{1}$. Now, $\Delta\left(y_{1}\right)$ and $\Delta_{*}\left(y_{1}\right) \in H^{1}(T)_{\mathcal{H}}$ are the objects of our study.

The results in [22] have obvious versions for complete pseudogroups of local isometries. In particular, when $T$ has an $\mathcal{H}$-invariant orientation, the top-dimensional invariant cohomology $H^{n}(T)_{\mathcal{H}}$ is non-trivial if and only if $\bigwedge^{m} \mathcal{C}$ is a trivial sheaf, which is equivalent to $\Delta_{*}\left(y_{1}\right)=0$.

4. The form $\Delta\left(y_{1}\right) \in \Omega^{1}(T)_{\mathcal{H}}$ when $\mathcal{H}$ preserves a parallelism. With the notation of Sect. 3, suppose $\mathcal{H}$ preserves a parallelism on $T$. Then, as a particular case of a foliation on a Riemannian manifold, there is a bigrading of $\Omega$ given by the fibers of $\pi_{\mathrm{b}}$ : If $\mathcal{V}$ is the vertical bundle of $\pi_{\mathrm{b}}$ and $\mathcal{Q}$ the orthogonal complement of $\mathcal{V}$, then

$$
\Omega^{u, v}=C^{\infty}\left(\bigwedge^{u} \mathcal{Q}^{*} \otimes \bigwedge^{v} \mathcal{V}^{*}\right), \quad u, v \in \mathbb{Z} .
$$

This bigrading is $\mathcal{H}$-invariant, and thus restricts to $\Omega_{\mathcal{H}}$. The de Rham derivative decomposes as $d=d_{0,1}+d_{1,0}+d_{2,-1}$, where each $d_{i, j}$ is bihomogeneous of bidegree $(i, j)$, and the usual formulae are satisfied (see e.g. [1]). Clearly $F^{k} \Omega_{\mathcal{H}}=\Omega_{\mathcal{H}}^{k, \cdot} \wedge \Omega_{\mathcal{H}}$, yielding canonical identities $\left(E_{0}, d_{0}\right) \equiv\left(\Omega_{\mathcal{H}}, d_{0,1}\right)$ and $\left(E_{1}, d_{1}\right) \equiv\left(H\left(\Omega_{\mathcal{H}}, d_{0,1}\right), d_{0,1 *}\right)$.

Let $\chi \in \Omega_{\mathcal{H}}^{0, m}$ be the characteristic form of the fibers of $\pi_{\mathrm{b}}$, where $m=$ $\operatorname{dim} \mathfrak{g}$. There is a form $\tau \in \Omega_{\mathcal{H}}^{0,1}$ such that, for any $\mathcal{H}$-invariant vector field $Y$ tangent to the $\mathcal{H}$-orbit closures,

$$
\theta_{Y} \chi \in-\tau(Y) \chi+F^{1} \Omega_{\mathcal{H}}^{m}
$$

Indeed, if $\mathcal{H}_{F}$ is the restriction of $\mathcal{H}$ to any $\mathcal{H}$-orbit closure $F$, then the Lie algebra $\mathcal{X}_{F}$ of $\mathcal{H}_{F}$-invariant vector fields on $F$ is isomorphic to $\mathfrak{g}$ by Salem's description, and the restriction $\tau_{F}$ of $\tau$ to $F$ is the trace of the adjoint representation of $\mathcal{X}_{F}$. So $\tau=0$ if and only if $\mathfrak{g}$ is unimodular. On the other hand, the mean curvature form $\kappa$ of the $\mathcal{H}$-orbit closures is in $\Omega_{\mathcal{H}}^{1,0}=F^{1} \Omega_{\mathcal{H}}^{1}$, and satisfies Rummler's formula

$$
\theta_{Z} \chi \in-\kappa(Z) \chi+F^{1} \Omega_{\mathcal{H}}^{m}
$$

for any $\mathcal{H}$-invariant vector field $Z$ orthogonal to the orbit closures. Also, with the notation of Sect. 3 for $V=C$, let $\kappa_{C}$ be the $\widehat{g}$-mean curvature of the fibers of the projection of $C$ to $T$.

Proposition 4.1. $\tau+\kappa=2 \pi \Delta\left(y_{1}\right)$.

P r o of. For any fixed subset $U \subset T / \overline{\mathcal{H}}$, let $C_{U}$ be the restriction of $C$ to $\pi_{\mathrm{b}}^{-1}(U)$. The statement of this result is a local property, thus it is enough to prove it on $\pi_{\mathrm{b}}^{-1}(U)$. Then, by Salem's description, we can assume $\pi_{\mathrm{b}}^{-1}(U)=$ $U \times G$, where $\overline{\mathcal{H}}$ is generated by the action of $G$, acting by left multiplication 
on itself and trivially on $U$. Thus $C_{U} \equiv U \times G \times \mathfrak{g}^{-} \equiv U \times \mathrm{T} G \subset \mathrm{T}(U \times G)$, and $C^{\infty}\left(C_{U}\right) \equiv C^{\infty}\left(U \times G, \mathfrak{g}^{-}\right)$, where the $\nabla$-parallel sections of $C_{U}$ are identified with the constant functions with values in $\mathfrak{g}^{-}$.

Let $X$ be a unitary section of $\wedge^{m} C_{U}$, which can be considered as a function on $U \times G$ with values in $\Lambda^{m} \mathfrak{g}^{-}$. By (9) and (10) we have

$$
\theta_{Y} X=(\tau+\kappa)(Y) X
$$

for any $\mathcal{H}$-invariant vector field $Y$ on $U \times G$.

The vertical bundle of $C_{U}$ can be canonically identified with the trivial bundle $C_{U} \times \mathfrak{g}^{-}$. Hence, using the notation of the proof of Proposition 2.1 for $V=C_{U}, \widehat{X}$ can be considered as a function on $C_{U}$ with values in $\bigwedge^{m} \mathfrak{g}^{-}$, which is clearly equal to the pull-back of $X$. So, as in (3),

$$
\widehat{\theta_{Y} X}=\theta_{\widetilde{Y}} \widehat{X}=\kappa_{C}(\tilde{Y}) \widehat{X}
$$

for any vector field $Y$ on $U \times G$, where $\widetilde{Y}$ is the horizontal lift of $Y$. Then the result follows from (11) and (12), and Proposition 2.1.

\section{Unimodularity of the structural Lie algebra}

THEOREM 5.1. If $\mathcal{H}$ is a complete pseudogroup of local isometries and $\mathfrak{g}$ its structural Lie algebra, then $\bar{\Delta}_{*}\left(y_{1}\right)=0$ if and only if $\mathfrak{g}$ is unimodular.

Proof. Since $\pi_{\infty}^{*}: E_{\infty}^{0,1}(\mathcal{H}) \rightarrow E_{\infty}^{0,1}(\widehat{\mathcal{H}})$ is injective and $\pi_{\infty}^{*} \bar{\Delta}(\mathcal{H})_{*}\left(y_{1}\right)=$ $\bar{\Delta}(\widehat{\mathcal{H}})_{*}\left(y_{1}\right)$, we can assume $\mathcal{H}$ preserves a parallelism.

With the above assumption, if $\bar{\Delta}_{*}\left(y_{1}\right)=0$ then $\Delta_{*}\left(y_{1}\right) \in F^{1} H^{1}(T)_{\mathcal{H}}$. So there is some $\mathcal{H}$-invariant function $f$ such that $\tau+\kappa+d f \in F^{1} \Omega_{\mathcal{H}}^{1}$. But $\kappa+d f \in F^{1} \Omega_{\mathcal{H}}^{1}$ because $d_{0,1}\left(\Omega_{\mathcal{H}}^{0}\right)=0$. Therefore $\tau \in \Omega_{\mathcal{H}}^{0,1} \cap F^{1} \Omega_{\mathcal{H}}^{1}=0$, and $\mathfrak{g}$ is unimodular.

If $\mathfrak{g}$ is unimodular, then $\tau=0$ and thus $\kappa \in F^{1} \Omega_{\mathcal{H}}^{1}$ represents $2 \pi \Delta_{*}\left(y_{1}\right)$. So $\Delta_{*}\left(y_{1}\right) \in F^{1} H^{1}(T)_{\mathcal{H}}$ and $\bar{\Delta}_{*}\left(y_{1}\right)=0$.

From Theorem 5.1, any vanishing result for $H^{1}(T)_{\mathcal{H}}$ yields the unimodularity of $\mathfrak{g}$. Such a result is proved e.g. in [3] by using Morse inequalities for pseudogroups of local isometries.

6. Minimality of the orbit closures when $\mathcal{H}$ preserves a parallelism. Assume $\mathcal{H}$ preserves a parallelism on $T$. With the notation of Sect. 4, let $\nu$ denote the normal bundle of the fibers of $\pi_{\mathrm{b}}$, which is canonically isomorphic to $\mathcal{Q}$, and let $C^{\infty}\left(\nu^{*} \otimes \mathcal{V}\right)_{\mathcal{H}}$ be the space of $\mathcal{H}$-invariant sections of the $\mathcal{H}$-vector bundle $\nu^{*} \otimes \mathcal{V}$. For $\sigma \in C^{\infty}\left(\nu^{*} \otimes \mathcal{V}\right)_{\mathcal{H}}$ and $x \in T$, let $\mathcal{Q}_{x}^{\sigma}=\left\{v+\sigma_{x}(\bar{v}): v \in \mathcal{Q}_{x}\right\}$, where $\bar{v}$ is the element in $\nu$ defined by $v$. Clearly $\mathcal{Q}^{\sigma}=\bigsqcup_{x \in T} \mathcal{Q}_{x}^{\sigma}$ is an $\mathcal{H}$-subbundle of the tangent bundle of $T$ which is complementary to $\mathcal{V}$. The correspondence $\sigma \mapsto \mathcal{Q}^{\sigma}$ defines a bijection between $C^{\infty}\left(\nu^{*} \otimes \mathcal{V}\right)_{\mathcal{H}}$ and the $\mathcal{H}$-bundles of tangent vectors which are 
complementary to $\mathcal{V}$. This correspondence depends on $\mathcal{Q}$, and thus on the given metric $g$. For each such $\sigma$, there is a unique $\mathcal{H}$-invariant metric $g^{\sigma}$ on $T$ such that $g$ and $g^{\sigma}$ induce the same metric on $T / \overline{\mathcal{H}}$ and define the same metric on $\mathcal{V}$, and so that the $g^{\sigma}$-orthogonal complement of $\mathcal{V}$ is $\mathcal{Q}^{\sigma}$. The $g^{\sigma}$-mean curvature form of the orbit closures will be denoted by $\kappa^{\sigma}$.

For any $\mathcal{H}$-invariant function $h$ on $T$, consider also the orthogonal sum $g^{h}$ of the restriction of $g$ to $\mathcal{Q}$ and the restriction of $\mathrm{e}^{h} g$ to $\mathcal{V}$. The $g^{h}$ will be said to be obtained from $g$ by a scalar change along the orbit closures.

THEOREM 6.1. Let $\mathcal{H}$ be a complete pseudogroup of local isometries which preserves a parallelism on a manifold $T$, and $\mathfrak{g}$ the structural Lie algebra. Then:

(i) If $\mathfrak{g}$ is unimodular, then $2 \pi \Delta_{*}\left(y_{1}\right)$ is the class of all possible mean curvature forms of the orbit closures for all the $\mathcal{H}$-invariant metrics on $T$. Thus, in this case, $\Delta_{*}\left(y_{1}\right)=0$ if and only if the orbit closures are minimal submanifolds for some $\mathcal{H}$-invariant metric.

(ii) If $\mathfrak{g}$ is not unimodular, then any element in $\Omega_{\mathcal{H}}^{1,0}$ is the mean curvature form of the orbit closures for some $\mathcal{H}$-invariant metric on $T$. Thus, in this case, the orbit closures are minimal submanifolds for some $\mathcal{H}$-invariant metric.

Thus $\Delta_{*}\left(y_{1}\right)=0$ if and only if $\mathfrak{g}$ is unimodular and the orbit closures are minimal submanifolds for some $\mathcal{H}$-invariant metric on $T$.

Pr o o f. Let $g$ be any $\mathcal{H}$-invariant metric. If $\mathfrak{g}$ is unimodular, then $\tau=0$ and $\kappa$ represents $2 \pi \Delta_{*}\left(y_{1}\right)$. On the other hand, any element in this class can be realized as the mean curvature form of the orbit closures for some metric obtained from $g$ by a scalar change along the orbit closures.

Suppose $\mathfrak{g}$ is not unimodular, and thus $\tau$ is a non-vanishing form. So there is an $\mathcal{H}$-invariant $\pi_{\mathrm{b}}$-vertical vector field $Y$ such that $\tau(Y)=1$. $(Y$ can be chosen to be $|\tau|^{-2}$ times the $g$-dual vector field of $\tau$.) Take any $\alpha \in \Omega_{\mathcal{H}}^{1,0}$ and define $\sigma \in C^{\infty}\left(\nu^{*} \otimes \mathcal{V}\right)_{\mathcal{H}}$ by $\sigma_{x}(\bar{v})=(\alpha+\kappa)_{x}(v) Y_{x}$ for any $x \in T$ and any tangent vector $v$ at $x$, and where $\bar{v}$ is the element defined by $v$ in $\nu_{x}$. Let $P^{\sigma}$ denote the $g^{\sigma}$-orthogonal projection of the tangent bundle of $T$ onto $\mathcal{V}$. It is easily verified that

$$
P^{\sigma}(v)=(\alpha+\kappa)_{x}(v) Y_{x} \quad \text { for } v \in \mathcal{Q}_{x} .
$$

For a local orientation of the fibers of $\pi_{\mathrm{b}}$, let $\chi$ and $\chi^{\sigma}$ be the corresponding characteristic forms for $g$ and $g^{\sigma}$, respectively. Let $Y_{1}, \ldots, Y_{m}$ be a local orthonormal frame of $\mathcal{V}$ such that $Y_{1}=Y /|Y|$. By $(13)$, for any $X \in C^{\infty}(\mathcal{Q})$ 
we have

$$
\begin{aligned}
\chi^{\sigma}\left(X \wedge Y_{2} \wedge \ldots \wedge Y_{m}\right) & =\chi\left(P^{\sigma}(X) \wedge Y_{2} \wedge \ldots \wedge Y_{m}\right) \\
= & \chi\left((\alpha+\kappa)(X) Y \wedge Y_{2} \wedge \ldots \wedge Y_{m}\right) \\
= & (\alpha+\kappa)(X)|Y| \\
\chi^{\sigma}\left(Y_{1} \wedge \ldots \wedge Y_{i-1}\right. & \left.\wedge X \wedge Y_{i+1} \wedge \ldots \wedge Y_{m}\right)=0 \\
\chi^{\sigma}\left(Y_{1}\right. & \left.\wedge \ldots \wedge Y_{m}\right)=1 .
\end{aligned}
$$

Thus

$$
\chi^{\sigma}=\chi+(\alpha+\kappa) \wedge i_{Y} \chi
$$

Therefore

$$
\begin{aligned}
d \chi^{\sigma} & =d\left(\chi+(\alpha+\kappa) \wedge i_{Y} \chi\right) \\
& =d \chi+d(\alpha+\kappa) \wedge i_{Y} \chi-(\alpha+\kappa) \wedge d i_{Y} \chi \\
& \in-\kappa \wedge \chi+d(\alpha+\kappa) \wedge i_{Y} \chi-(\alpha+\kappa) \wedge\left(\theta_{Y}-i_{Y} d\right) \chi+F^{2} \Omega_{\mathcal{H}}^{m} \\
& =-\kappa \wedge \chi-(\alpha+\kappa) \wedge \theta_{Y} \chi+F^{2} \Omega_{\mathcal{H}}^{m} .
\end{aligned}
$$

But $\theta_{Y} \chi=-\chi$ by the definition of $\tau$ and the choice of $Y$. So

$$
d \chi^{\sigma} \in-\alpha \wedge \chi+F^{2} \Omega_{\mathcal{H}}=-\alpha \wedge \chi^{\sigma}+F^{2} \Omega_{\mathcal{H}},
$$

yielding $\kappa^{\sigma}=\alpha$ by Rummler's mean curvature formula, and the proof is finished.

ExAmple 6.2 (Y. Carrière). The affine Lie group $A$ can be identified with $\mathbb{R}^{2}$ with the group structure given by $(t, s)\left(t^{\prime}, s^{\prime}\right)=\left(t+t^{\prime}, \lambda^{t} s^{\prime}+s\right)$ for any fixed $\lambda>1$. Consider the pseudogroup generated by the left action of the closed subgroup $K=\mathbb{Z} \times \mathbb{R} \subset A$ on $A$. Clearly $K \backslash A \equiv \mathbb{S}^{1}$, the structural Lie algebra is abelian, and we have $H^{2}(A)_{K}=0$ [4]. Hence $\bar{\Delta}_{*}\left(y_{1}\right)=0$ and $\Delta_{*}\left(y_{1}\right) \neq 0$. Therefore there is no $K$-invariant metric on $A$ such that the right translates of $K$ are minimal submanifolds.

E. Macías and E. Sanmartín have proved the following [14]: If $H$ is a Lie subgroup of a Lie group $G$, and $H_{0}$ the connected component of $H$ which contains the identity element, then the right translates of $H$ are minimal submanifolds for some metric on $G$. Moreover, from the proof in [14] it can be easily seen that the above metric can be chosen to be invariant by the left action of $H_{0}$. So the non-triviality of $\Delta_{*}\left(y_{1}\right)$ in Example 6.2 depends on the disconnectedness of $K$. From Theorems 5.1 and 6.1 , we get the following generalization of the results of [14], where $\Delta_{*}\left(y_{1}\right)$ and $\bar{\Delta}_{*}\left(y_{1}\right)$ are defined by the pseudogroup generated by the left action of $H$ on $G$.

COROllary 6.3. With the above notation, we have:

(i) $\bar{\Delta}_{*}\left(y_{1}\right)=0$ if and only if $H$ is unimodular. 
(ii) If $H$ is unimodular, then $\Delta_{*}\left(y_{1}\right)=0$ if and only if the right translates of $H$ are minimal submanifolds for some metric on $G$ which is invariant by the left $H$-action.

(iii) If $H$ is not unimodular, then the right translates of $H$ are minimal submanifolds for some metric on $G$ which is invariant by the left $H$-action.

If $\mathcal{H}$ is not required to preserve a parallelism on $T$, then Theorems 5.1 and 6.1 have the following consequence by considering $\widehat{\mathcal{H}}$.

Corollary 6.4. If $\mathcal{H}$ is a complete pseudogroup of local isometries, and $\mathfrak{g}$ its structural Lie algebra, then:

(i) If $\mathfrak{g}$ is unimodular, then $\Delta_{*}\left(y_{1}\right)=0$ if and only if the $\widehat{\mathcal{H}}$-orbit closures are minimal submanifolds for some $\widehat{\mathcal{H}}$-invariant metric.

(ii) If $\mathfrak{g}$ is not unimodular, then the $\widehat{\mathcal{H}}$-orbit closures are minimal submanifolds for some $\widehat{\mathcal{H}}$-invariant metric.

Thus $\Delta_{*}\left(y_{1}\right)=0$ if and only if $\mathfrak{g}$ is unimodular and the $\widehat{\mathcal{H}}$-orbit closures are minimal submanifolds for some $\widehat{\mathcal{H}}$-invariant metric.

7. Application to Riemannian foliations. Let $\mathcal{F}$ and $M$ be as in Sect. 1. Let $\mathcal{H}$ be the representative of the holonomy pseudogroup of $\mathcal{F}$ canonically defined on a manifold $T$ by some regular covering of $M$ (see e.g. $[9,10])$. Then any fixed transverse Riemannian structure of $\mathcal{F}$ canonically corresponds to an $\mathcal{H}$-invariant metric on $T$ so that $\mathcal{H}$ is a complete pseudogroup of local isometries, and there are canonical isomorphisms

$$
\begin{aligned}
& \Omega^{\cdot}(T)_{\mathcal{H}} \cong \Omega^{\cdot}(M / \mathcal{F}), \\
& H^{\cdot}(T)_{\mathcal{H}} \cong H^{\cdot}(M / \mathcal{F}) .
\end{aligned}
$$

More precisely, let $\left\{\left(U_{i}, f_{i}\right)\right\}$ be a regular covering of $M$. The restriction of $\mathcal{F}$ to each $U_{i}$ is given by the submersion $f_{i}$ of $U_{i}$ onto some manifold $T_{i}$. The regularity of this covering means that there are well defined diffeomorphisms $h_{i j}: f_{i}\left(U_{i} \cap U_{j}\right) \rightarrow f_{j}\left(U_{i} \cap U_{j}\right)$ such that $h_{i j} f_{i}=f_{j}$ on $U_{i} \cap U_{j}$. Then $T=\bigsqcup_{i} T_{i}$ and $\mathcal{H}$ is generated by the $h_{i j}$. The metric on $T$ is determined by requiring the $f_{i}$ to be Riemannian submersions. The isomorphism in (15) is given by $\alpha \mapsto \alpha^{\prime}$, where $f_{i}^{*}\left(\left.\alpha\right|_{T_{i}}\right)=\left.\alpha^{\prime}\right|_{U_{i}}$. Moreover, each $f_{i}^{*} C(\mathcal{H})$ is canonically isomorphic to the restriction of $C(\mathcal{F})$ to $U_{i}$, so the classes $\Delta(\mathcal{H})_{*}\left(y_{1}\right)$ and $\Delta(\mathcal{F})_{*}\left(y_{1}\right)$ correspond to each other by $(16)$.

Proof of The or em 1.1. We can suppose $\mathcal{F}$ is transversely parallelizable by using $\widehat{\mathcal{F}}$ in a standard way. Then $M / \overline{\mathcal{F}} \equiv T / \overline{\mathcal{H}}$ is a manifold, and the canonical map $\pi_{\mathrm{b}}: M \rightarrow M / \overline{\mathcal{F}}$ a fiber bundle projection whose fibers define thus a foliation $\overline{\mathcal{F}}$. We can suppose the metric on $M$ is chosen so that the leaf closures are minimal submanifolds. On any fixed $U_{i}$, the vector bundles $\mathrm{T} \mathcal{F}$, $\mathrm{T} \overline{\mathcal{F}}$ and $\mathrm{T} \mathcal{F}^{\perp} \cap \mathrm{T} \overline{\mathcal{F}}$ are orientable, and take the unitary sections $X, X^{\prime}$ and 
$X^{\prime \prime}$ on $U_{i}$ defining respective positive orientations of $\bigwedge^{p} \mathrm{~T} \mathcal{F}, \bigwedge^{p+m} \mathrm{~T} \overline{\mathcal{F}}$ and $\bigwedge^{m}\left(\mathrm{~T} \mathcal{F}^{\perp} \cap \mathrm{T} \overline{\mathcal{F}}\right.$ ), where $p=\operatorname{dim} \mathcal{F}$ (thus $p+m=\operatorname{dim} \overline{\mathcal{F}}$ ). The orientations can be chosen so that $X^{\prime}=X \wedge X^{\prime \prime}$. Let $Y$ be an infinitesimal transformation of $\mathcal{F}$ which is orthogonal to the leaves. Since $X \wedge \wedge^{+} C^{\infty}(\mathrm{T} \mathcal{F})=0$, we get $X \wedge \theta_{Y} X^{\prime \prime}=\left(2 \pi f_{i}^{*} \Delta(\mathcal{F})\left(y_{1}\right)\right)(Y) X^{\prime}$ by (11) and Proposition 4.1. Hence

$$
\theta_{Y} X^{\prime}=\left(\kappa_{\mathcal{F}}+2 \pi f_{i}^{*} \Delta(\mathcal{H})\left(y_{1}\right)\right)(Y) X^{\prime}
$$

by Rummler's formula, where $\kappa_{\mathcal{F}}$ is the mean curvature form of the leaves. On the one hand, if $Y$ is orthogonal to $\overline{\mathcal{F}}$, then $\theta_{Y} X^{\prime}=0$ because the leaves of $\overline{\mathcal{F}}$ are minimal submanifolds, and we get $\left(\kappa_{\mathcal{F}}+2 \pi \Delta(\mathcal{F})\left(y_{1}\right)\right)(Y)=0$ on $M$. On the other hand, if $Y$ is tangent to $\overline{\mathcal{F}}$, then $\theta_{Y} X^{\prime}=-\operatorname{div}_{\mathrm{b}}(Y) X^{\prime}$ where $\operatorname{div}_{\mathrm{b}}$ denotes the divergence on the fibers of $\pi_{\mathrm{b}}$, and we get

$$
\left(\kappa_{\mathcal{F}}+2 \pi \Delta(\mathcal{F})\left(y_{1}\right)\right)(Y)=-\operatorname{div}_{\mathrm{b}}(Y)
$$

on $M$. Therefore the function $\left(\kappa_{\mathcal{F}}+2 \pi \Delta(\mathcal{F})\left(y_{1}\right)\right)(Y)$ has trivial integral on the fibers of $\pi_{\mathrm{b}}$ for any infinitesimal transformation $Y$ of $\mathcal{F}$ on $M$. This implies that $\kappa_{\mathcal{F}}+2 \pi \Delta(\mathcal{F})\left(y_{1}\right)$ has trivial basic component [2]; i.e. the basic component of $\kappa_{\mathcal{F}}$ is equal to $-2 \pi \Delta(\mathcal{F})\left(y_{1}\right)$, and the result follows.

The other results in Sect. 1 now follow directly from Theorem 1.1 and the results in Sects. 5 and 6.

\section{References}

[1] J. A. Álvarez López, A finiteness theorem for the spectral sequence of a Riemannian foliation, Illinois J. Math. 33 (1989), 79-92.

[2] - , The basic component of the mean curvature of Riemannian foliations, Ann. Global Anal. Geom. 10 (1992), 179-194.

[3] -, Morse inequalities for pseudogroups of local isometries, J. Differential Geom. 37 (1993), 603-638.

[4] Y. Carrière, Flots riemanniens, Astérisque 116 (1984), 31-52.

[5] D. Domínguez, Finiteness and tenseness theorems for Riemannian foliations, preprint, 1994.

[6] A. El Kacimi-Alaoui et G. Hector, Décomposition de Hodge sur l'espace des feuilles d'un feuilletage riemannien, C. R. Acad. Sci. Paris 298 (1984), 289-292.

[7] A. El Kacimi-Alaoui, G. Hector et V. Sergiescu, La cohomologie basique d'un feuilletage Riemannien est de dimension finie, Math. Z. 188 (1985), 593-599.

[8] A. El Kacimi-Alaoui and M. Nicolau, On the topological invariance of the basic cohomology, Math. Ann. 293 (1993), 627-634.

[9] A. Haefliger, Some remarks on foliations with minimal leaves, J. Differential Geom. 15 (1980), 269-384.

[10] -, Pseudogroups of local isometries, in: Differential Geometry, Proc. Conf. Santiago de Compostela 1984, L. A. Cordero (ed.), Pitman, 1984, 174-197.

[11] - , Leaf Closures in Riemannian Foliations, in: A Fête on Topology, Academic Press, New York, 1988, 3-32. 
[12] F. Kamber and P. Tondeur, Foliated Bundles and Characteristic Classes, Lecture Notes in Math. 494, Springer, 1975.

[13] —, - De Rham-Hodge theory for Riemannian foliations, Math. Ann. 277 (1987), 415-431.

[14] E. Macías and E. Sanmartín, Minimal foliations on Lie groups, Indag. Math. 3 (1992), 41-46.

[15] X. Masa, Duality and minimality in Riemannian foliations, Comment. Math. Helv. 67 (1992), 17-27.

[16] P. Molino, Géométrie globale des feuilletages riemanniens, Nederl. Akad. Wetensch. Proc. A1 85 (1982), 45-76.

[17] —, Riemannian Foliations, Progr. Math. 73, Birkhäuser, Boston, 1988.

[18] P. Molino et V. Sergiescu, Deux remarques sur les flots riemanniens, Manuscripta Math. 51 (1985), 145-161.

[19] B. L. Reinhart, Foliated manifolds with bundle-like metrics, Ann. of Math. 69 (1959), 119-132.

[20] H. Rummler, Quelques notions simples en géométrie riemannienne et leurs applications aux feuilletages compacts, Comment. Math. Helv. 54 (1979), 224-239.

[21] E. Salem, Riemannian foliations and pseudogroups of isometries, in: Riemannian Foliations, Progr. Math. 73, Birkhäuser, Boston, 1988

[22] V. Sergiescu, Cohomologie basique et dualité des feuilletages riemanniens, Ann. Inst. Fourier (Grenoble) 35 (1985), 137-158

[23] A. Verona, A de Rham type theorem, Proc. Amer. Math. Soc. 104 (1988), 300-302.

Universidade de Santiago de Compostela

Facultade de Ciencias

27071 Lugo, Spain

E-mail: xtzzl002@seinl.usc.es 